\title{
КОНЦЕПТУАЛЬНАЯ МОДЕЛЬ ИНТЕЛЛЕКТУАЛЬНОЙ ОБРАЗОВАТЕЛЬНОЙ ЭКОСИСТЕМЫ
}

\section{A CONCEPTUAL MODEL OF THE INTELLIGENT EDUCATIONAL ECOSYSTEM}

\author{
Д.М. Оболенский, В.И. Шевченко \\ D.M. Obolensky, V.I. Shevchenko
}

\author{
Севастопольский государственный университет, \\ Россия, 299053, г. Севастополь, ул. Университетская, 33 \\ Sevastopol State University, 33 Universitetskaya St, Sevastopol, 299053, Russia \\ E-mail: denismaster@outlook.com, VIShevchenko@sevsu.ru
}

\begin{abstract}
Аннотация
В данной статье авторы рассматривают современные подходы, применяемые в дистанционном образовании, определяют проблемы дистанционного обучения, в частности проблему обратной связи, релевантности, поиска необходимых курсов, а также актуальности курсов с учетом развития компетентностной парадигмы получения знаний. В ходе работы исследуются идеи, способные улучшить обратную связь и качество получаемых знаний, персонализировать изучаемый материал, а также учитывать актуальность изучаемых курсов на рынке труда. Изучается идея индивидуальных образовательных траекторий, формируемых в зависимости от индивидуальных навыков, возможностей студента, а также его целей. Предложена концептуальная модель интеллектуальной образовательной экосистемы, описывается ее структура и метрики. На основе данной концепции планируется разработка СППР для построения персональных рекомендаций в дистанционном образовании с использованием современных методов машинного обучения и искусственного интеллекта.
\end{abstract}

\begin{abstract}
In this article, the authors explore modern approaches used in distance education, defines the problems of distance learning, such as the problem of feedback, relevance, the search for the necessary courses, as well as the importance of courses, taking into account the competency-based paradigm of knowledge acquisition. During the research, the author examines ideas that can improve feedback and the quality of knowledge gained, personalize the subjects studied. The author suggests also to use job and CV datasets to find important statistic data required for these improvements. The idea of individual educational paths is being studied, which are formed depending on individual skills, student cognitive abilities as well as his goals. A conceptual model of the intellectual educational ecosystem is proposed, its structure and metrics are described. Based on this concept, the authors planned to develop a DSS for buildling personal recommendations in distance education using modern methods of machine learning and artificial intelligence.
\end{abstract}

Ключевые слова: индивидуальная образовательная траектория, образовательная экосистема, персонализация, дистанционное образование.

Keywords: individual educational path, educational ecosystem, personalization, distance education.

\section{Введение}

Цифровизация образовательной деятельности является одной из тенденций современного общества. Понятия, связанные с технологиями дистанционного образования (ДОТ) и электронного обучения (ЭО), закреплены на законодательном уровне в Федеральном законе от 29.12.2012 № 273-Ф3 «Об образовании в Российской Федерации» [ФЗ РФ, 2012]. Специфика образовательной деятельности высших школ предполагает огромные 
объемы информации, мобильность обучающихся, увеличение доли самостоятельной работы [Козлова и др., 2017]. В этом случае применение ЭО и ДОТ является существенным преимуществом.

Однако онлайн-обучение имеет также свои недостатки. Помимо отсутствия мотивации и низкой статистики проходимости курсов, можно отметить отсутствие индивидуализации материала курса для отдельного студента. Темп подачи материала задается автором, и не всегда может подходить под индивидуальные когнитивные способности студентов. Затруднено получения обратной связи от студентов, так как многие курсы рассчитаны на широкие массы. Таким образом, становится сложнее произвести оценку эффективности той или иной технологии обучения, определить факторы, влияющие на обучение, оценить квалификацию преподавателей [ФЗ РФ, 2012; Козлова и др., 2017].

Для решения поставленных проблем современная система образования предлагает концепцию «индивидуальной образовательной траектории обучения». Основными идеями, лежащими в основе индивидуальных образовательных траекторий (ИОТ), лежат понятие адаптивного, модульного и персонализированного образования. Адаптивность подразумевает наличие обратной связи между студентом и преподавателем, а также систему мотивации. Модульность подачи учебных материалов позволяет кластеризировать знания и практические умения по вырабатываемым навыкам. Персонализация позволяет сформировать индивидуальную последовательность изучения дисциплин для студента на основе заданных целей обучения, а также использовать наиболее подходящие по форме изложения материалы [Козлова и др., 2017; Мащенко и др., 2017].

Однако существующие на сегодняшний день методы и модели формирования индивидуальных образовательных траекторий не в полной мере учитывают особенности формирования компетентностного профиля современного эффективного специалиста, а также запросы работодателей для решения текущих и будущих задач бизнеса. В связи с чем актуальной является задача разработки на основе компьютерных моделей и современных информационных технологий новых моделей формирования компетентностного профиля на основе индивидуальных образовательных траекторий с применением ЭО и ДОТ [Гримута, Шевченко, 2019].

В рамках данной статьи предлагается концептуальная модель интеллектуальной образовательной экосистемы (ИОЭ). Данная экосистема должна учитывать различные виды образовательных материалов, предоставлять актуальные и релевантные рекомендации с учетом потребностей как пользователя, так и будущих работодателей.

\section{Подходы к моделированию интеллектуальной образовательной экосистемы}

С целью более чёткого позиционирования тематики работы, для построения концептуальной модели интеллектуальной образовательной экосистемы необходимо рассмотреть уже существующие подходы к организации образовательных траекторий и модели представления знаний в данном направлении.

Модель знаний, согласно работе [Любченко, 2011], можно представить в виде оверлейной модели в виде сети концептов. Данные концепты связаны друг с другом по принципу семантической сети или графа. Наложение данной модели на компетентностную парадигму позволяет различным образом сформировать подграфы знаний, соответствующих различным компетенциям. Это также позволяет формировать, дополнять, развивать наборы знаний, входящие в компетенции, формировать граф навыков специальностей. Пример модели концептов представлен на рисунке 1.

Эффективный способ формирования индивидуальных траекторий - дифференцированное обучение [Су, 2008]. Авторы учебного плана и преподаватели работают вместе над составлением нескольких предопределенных траекторий развития, а студент вправе выбрать наиболее подходящую для него. Еще одним способом формирования такой индивидуальной траектории является кластеризация групп обучающихся на основании различных метрик [Чен, Ду, 2008]. Данный подход наиболее прост в реализации, а также очень схож с традиционным, линейным режимом обучения [Кравец и др., 2017]. 


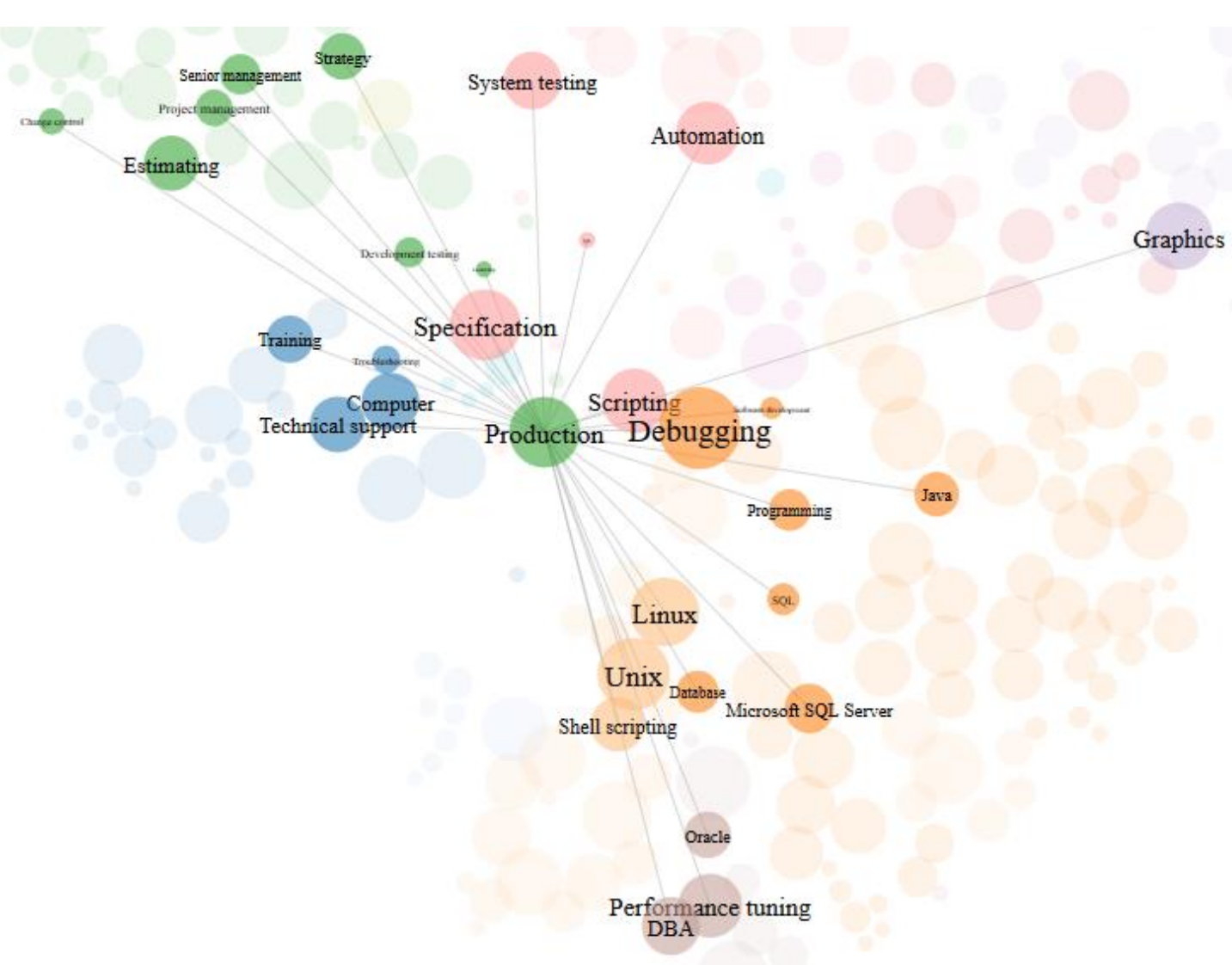

Рис. 1. Пример графа концептов

Fig. 1. An example of the concept graph

Метод деревьев решений позволяет направлять студента по тому или иному пути в зависимости от результатов оценки его знаний. Авторы курса заранее должны предложить методику оценивания знаний, порядок выполнения тестов, а также предопределить дерево решений [Чен, 2008]. Индивидуальная образовательная траектория формируется в таком случае динамически, на основании результатов оценивания. Очевидно, что в таком методе важную роль играет сама методика оценивания. В работе Афанасьева, Войта [Афанасьев, Войт, 2013] предлагается использование нечётких карт Кохонена для оценки и диагностики знаний и умений студентов. В работе Солдаткина [Солдаткин, 2013] предлагается автоматизированный способ генерации тестовых заданий на основе композиции нечетких множеств. Вероятностные модели, представленные в работе Серебровского и др. [Серебровский и др., 2013], позволяют представить процесс дистанционного обучения как вероятностную динамическую систему. Совокупность состояний, последовательная смена данных состояний, а также вероятности переходов между этими состояниями позволяет динамически формировать индивидуальные траектории для студентов на основе различных метрик [Лин и др., 2013].

Графовая модель, согласно работам Глущенко, Любченко [Глущенко, 2009; Любченко, 2011] также позволяет учитывать особенности студента, его знаний, а также его способности учиться. Построение индивидуальной траектории сводится к формированию множества возможных вариантов дальнейших действий. Например, система может предложить повторить знания по какому-либо материалу. В итоге формируется нелинейный граф прохождения изучения разделов учебного материала. Данная модель является наиболее сложной в реализации. Построение графа знаний и графа учебных материалов позволяет также формировать дорожные карты развития специалиста. Рассмотренные модели можно обобщить и скомбинировать, построив математическую модель ИОЭ. 


\section{Формальное описание конщептуальной модели интеллектуальной образовательной экосистемы}

Для формализации описания модели ИОЭ необходимо ввести ряд математических понятий. Пусть задано множество всех направлений подготовки $S P$ :

$$
S P=\left\{s p_{i}\right\}_{i=1}^{\|S P\|}
$$

Под направлением подготовки далее подразумевается определенная область знаний, сфера деятельности и т. д.

Также задано множество всех компетенций $S$ :

$$
S=\left\{S_{j}\right\}_{j=1}^{\|S\|}
$$

Также задано глобальное множество различных дисциплин (курсов) $C$ :

$$
C=\left\{c_{k}\right\}_{k=1}^{\|C\|}
$$

Каждому направлению подготовки можно поставить в соответствие множество компетенций этого направления. Таким образом, зададим бинарное отношение [Лаврентьев, Шабат, 1972] между этими двумя множествами в виде функции SSP:

$$
\forall s p \in S P, S S P(s p): S P \rightarrow S^{\prime}: S^{\prime} \subseteq S
$$

С другой стороны, при изучении каждой дисциплины осваиваются определенные компетенции. Это значит, что каждой дисциплине можно поставить в соответствие некоторое множество компетенций. Зададим бинарное отношение между этими двумя множествами в виде функции $S C$ :

$$
\forall c \in C, S C(c): C \rightarrow S^{\prime \prime}: S^{\prime \prime} \subseteq S
$$

Для дальнейших рассуждений, при помощи функций $S P$ и $S C$ определим множество дисциплин для данного направления подготовки в зависимости от строгости рассуждений. Определим функцию $\operatorname{CSP}(s p)$, которая вернет множество дисциплин, релевантных для выбранного направления.

Нестрого говоря, дисциплина $c$ релевантна выбранной специальности $s p$, если пересечение множеств $S S P(s p)$ и $S C(c)$ - непустое множество:

$$
\operatorname{CSP}(s p)=\{c \in C \mid S C(c) \cap \operatorname{SSP}(s p) \neq \varnothing\} .
$$

Строго говоря, курс $c$ релевантен выбранному направлению $s p$, если множество $S C(c)$ является подмножеством $S S P(s p)$ :

$$
\operatorname{CSP}(s p)=\{c \in C \mid S C(c) \subseteq \operatorname{SSP}(s p)\} .
$$

Выбор строгости рассуждений $(6,7)$ влияет лишь на мощность результирующего множества $\operatorname{CSP}(s p)$.

На практике дисциплины изучаются в определенном порядке. Например, для изучения курса по базам данных необходимо сперва изучить курсы по базовой математике, информатике и реляционной алгебре. Некоторые дисциплины могут дублировать информацию друг друга, например, курсы различных авторов или на различных образовательных платформах. 
Пусть задано бинарное отношение частичного упорядочивания $\preccurlyeq$, связывающее два произвольных курса. Если для произвольных дисциплин $c_{i}$ и $c_{j}$ выполняется данное бинарное отношение $c_{i} \preccurlyeq c_{j}$, то это означает, что для изучения курса $c_{i}$ необходимо изучить предварительно курс $c_{j}$.

Если для произвольных дисциплин $c_{i}$ и $c_{j}$ выполняются соотношения $c_{i} \preccurlyeq c_{j}$ и $c_{j} \preccurlyeq c_{i}$, то это означает, что дисциплины $c_{i}$ ис $c_{j}$ эквивалентны.

Если для произвольных дисциплин $c_{i}$ и $c_{j}$ не выполняются соотношения $c_{i} \preccurlyeq c_{j}$ или $c_{j} \preccurlyeq c_{i}$, то это означает, что дисциплины $c_{i}$ ис $c_{j}$ несравнимы, т. е нельзя сказать, в каком порядке их следует изучать.

Таким образом, для выбранной специальности $s p$, задав функцию $\operatorname{CSP}(s p)$ и бинарное отношение $\preccurlyeq$, можно сформировать (8) направленный ациклический граф [Туласираман, Свами, 1992] изучения данных дисциплин $D A C G(s p)$ :

$$
\operatorname{DACG}(s p)=<\operatorname{CSP}(s p), \operatorname{ESP}(s p)>\text {, }
$$

где множество $\operatorname{ESP}(s p)$ задано (9) следующим образом:

$$
\operatorname{ESP}(s p)=\left\{\left(c_{i}, c_{j}\right) \mid c_{i} \in \operatorname{CSP}(s p), c_{j} \in \operatorname{CSP}(s p), c_{i} \preccurlyeq c_{j}\right\}
$$

В качестве вершин данного графа для выбранного направления подготовки $s p$ мы используем множество $C S P(s p)$. В качестве рёбер графа используется множество $E S P(s p)$, т. е. такие пары $\left(c_{i}, c_{j}\right)$, состоящие из элементов множества $\operatorname{CSP}(s p)$, для которых выполняется заданное бинарное отношение $\preccurlyeq$.

Пример данного графа $D A C G(s p)$ представлен на рисунке 2.

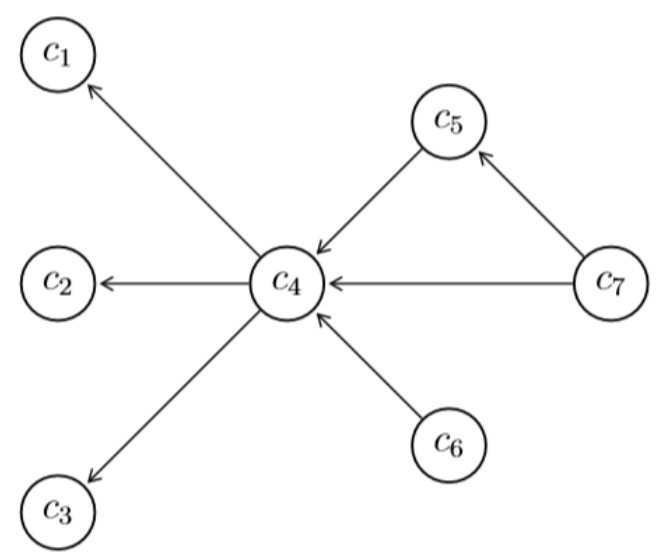

Рис. 2. Пример графа дисциплин

Fig 2. An example of the graph DACG

Любой студент, получающий образование и использующий для этого дистанционные онлайн-курсы, обладает различными когнитивными способностями, а также различными целями в жизни. С другой стороны, дисциплины также обладают различными характеристиками, равно как и развиваемые ею компетенции. В связи с этим, использование отношения $\preccurlyeq$ является недостаточным для полноценного описания пути развития человека с использованием графа $D A C G(s p)$. $\operatorname{ESP}(s p)$ :

Введем специальную матрицу весов $W(u, s p)$, задающую вес каждого ребра из

$$
W(u, s p)=\left\{w_{i, j}\right\}_{i=1, j=1}^{\|C S P(s p)\|}
$$


Данная матрица используется совместно с бинарным отношением $\preccurlyeq$. Сформируем взвешенный направленный ациклический граф $W D A C G(s p)$ :

$$
W D A C G(s p)=<\operatorname{CSP}(s p), \operatorname{ESP}(s p), W>\text {. }
$$

Пример данного графа $W D A C G(s p)$ представлен на рисунке 3.

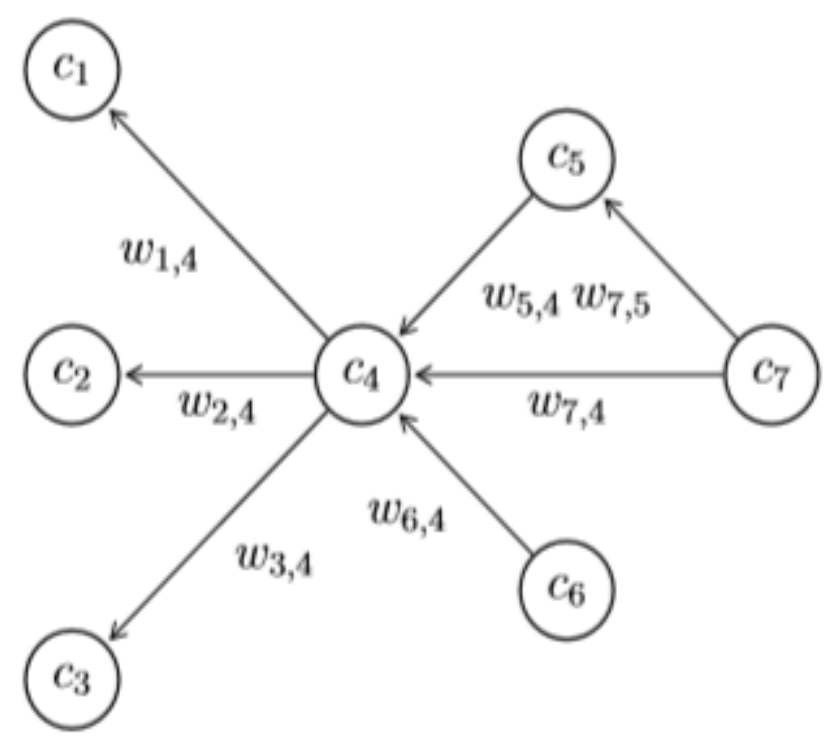

Рис. 3. Пример взвешенного графа дисциплин

Fig 3. An example of the weighted graph WDACG

Для дальнейшей формализации необходимо учитывать информацию о пользовательских компетенциях. Пусть задано множество всех пользователей интеллектуальной образовательной экосистемы $U$ :

$$
U=\left\{u_{l}\right\}_{l=1}^{\|U\|} .
$$

Каждый пользователь может указать, какими навыками он уже обладает на данный момент, а также какими компетенциями он бы хотел обладать в дальнейшем.

Для выбранного пользователя $u$ определим функцию $S_{\text {start }}(u)$, которая будет использоваться для получения множества уже обладаемых компетенций:

$$
S_{\text {start }}(u): U \rightarrow S_{\text {start }}^{u}: S_{\text {start }}^{u} \subseteq S \text {. }
$$

Также определим функцию $S_{\text {target }}(u)$, которая будет использоваться для получения множества желаемых компетенций:

$$
S_{\text {target }}(u): U \rightarrow S_{\text {target }}^{u}: S_{\text {target }}^{u} \subseteq S \text {. }
$$

Введем дополнительные обозначения $S_{\text {start }}(u, s p)$ и $S_{\text {target }}(u, s p)$ для ограничения множеств уже полученных и целевых навыков только теми компетенциями, которые релевантны выбранному направлению подготовки $s p$ :

$$
\begin{aligned}
S_{\text {start }}(u, s p) & =\left\{s \in S_{\text {start }}(u) \mid s \in S S P(s p)\right\}, \\
S_{\text {target }}(u, s p) & =\left\{s \in S_{\text {target }}(u) \mid s \in S S P(s p)\right\} .
\end{aligned}
$$


На основании данных обозначений и определений $(13,14)$ сформируем $(15)$ множество целевых дисциплин $C_{\text {target }}(u, s p)$, т. е. таких курсов, которые позволят пользователю $u$ сформировать желаемые компетенции:

$$
C_{\text {target }}(u, s p)=\left\{c \in C S P(s p) \mid S C(c) \subset S_{\text {target }}(u, s p)\right\}
$$

Пример множества целевых дисциплин показан на рисунке 4.

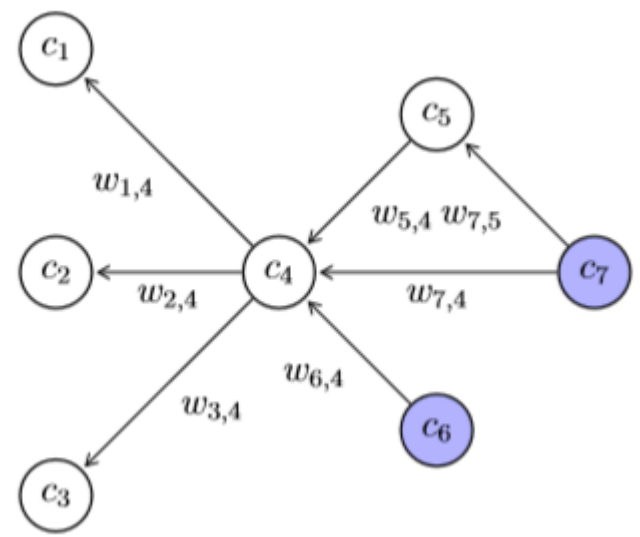

Рис. 4. Пример множества целевых дисциплин Fig 4. An example of the target skills set

Сформируем множество уже нерелевантных для пользователя дисциплин $C_{\text {start }}(u, s p)$, т. е. таких курсов, которые развивают те компетенции, которыми пользователь $u$ уже обладает на данный момент времени

$$
C_{\text {start }}(u, s p)=\left\{c \in C S P(s p) \mid S C(c) \subset S_{\text {start }}(u, s p)\right\}
$$

Пример множества уже изученных дисциплин показан на рисунке 5.

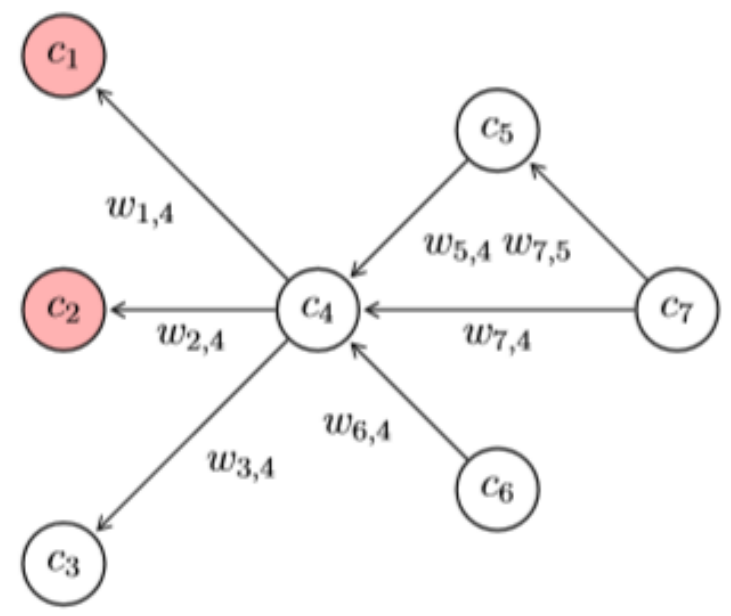

Рис. 5. Пример множества уже изученных дисциплин

Fig. 5. An example of the achieved skills set

Так как задано бинарное отношение $\preccurlyeq$, определяющее взаимосвязь двух курсов, а также множества $C_{\text {start }}(u, s p)$ и $C_{\text {target }}(u, s p)$, мы можем сформировать множество промежуточных дисциплин $C_{\text {path }}(u, s p)$. Для этого необходимо выбрать такие дисциплины, объединение множеств компетенций которых включает в себя множество $S_{\text {target }}(u, s p)$, при этом 
дисциплины требуются для изучения курсов из множества $C_{\text {target }}(u, s p)$, но при этом не принадлежат множеству $C_{\text {start }}(u, s p)$, а также не эквивалентны дисциплинам этого множества:

$$
\begin{array}{r}
C_{\text {path }}(u, s p)=\left\{c \in C S P(s p) \mid S_{\text {target }}(u, s p) \subseteq \cup S C(c),\right. \\
\left.\forall t c \in C_{\text {target }}(u, s p) t c \preccurlyeq c, \forall s c \in C_{\text {start }}(u, s p) c \neq s c \vee c \preccurlyeq s c\right\}
\end{array}
$$

Множество возможных дисциплин для изучения показано на рисунке 6.

Для каждого конечного пользователя множества $C_{\text {start }}(u, s p), C_{\text {target }}(u, s p)$, $C_{\text {path }}(u, s p)$ будут различными. Это дает возможность персонализировать порядок изучения дисциплин, чтобы он был оптимальным для обучающегося. Индивидуальные особенности учитываются в матрице весов $W(u, s p)(10)$, и могут быть настроены лицом, принимающим решение.

Для выбранного графа $W D A C G(s p)$ и выбранного пользователя $u$ получены начальные вершины $C_{\text {start }}(u, s p)$ и конечные вершины $C_{\text {target }}(u, s p)$.

Пусть $e_{i, j}$ - некоторое ребро графа $W D A C G(s p)$, соединяющее $c_{i}$ и $c_{j}$. Определим множество путей из множества начальных вершин $C_{\text {start }}(u, s p)$ в конечные $C_{\text {target }}(u, s p)$ в графе $W D A C G(s p)$. Для этого зададим функцию $P\left(u, s p, c_{\text {start }}, c_{\text {target }}\right)$ :

$$
\begin{array}{r}
P\left(u, s p, c_{\text {start }}, c_{\text {target }}\right)=\left(c_{1}, c_{2}, \ldots c_{n}\right), c_{1}=c_{\text {start }}, c_{n}=c_{\text {target }}, \\
c_{\text {start }} \in C_{\text {start }}(u, s p), c_{\text {target }} \in C_{\text {target }}(u, s p), \\
c_{i} \in C_{\text {path }}(u, s p) i=2 \ldots n-1
\end{array}
$$

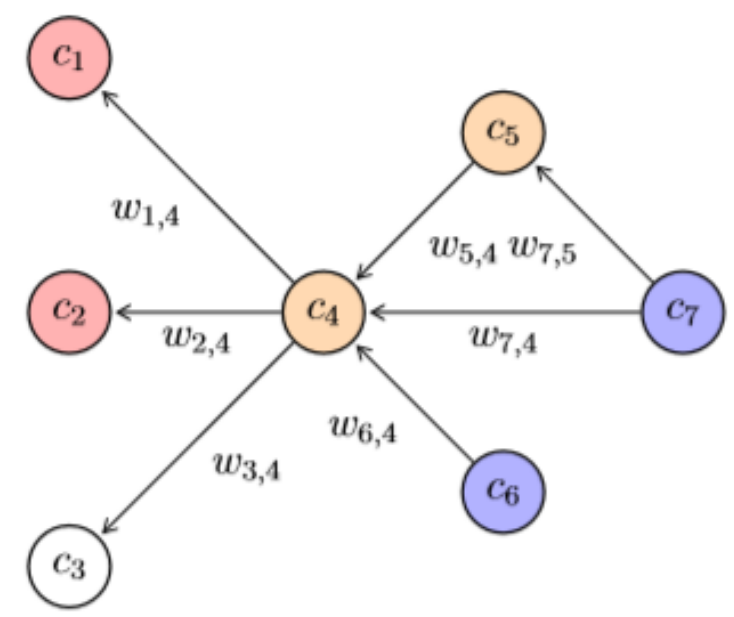

Рис. 6. Пример множества возможных дисциплин

Fig 6. An example of the possible skills set

Длина пути len $(P)$ на графе $W D A C G(s p)$ для пользователя $u$ определяет оптимальную последовательность дисциплин, необходимых для достижения целевых навыков $S_{\text {target }}(u, s p)$, например, оптимальную по времени изучения или наиболее подходящую в соответствии с познавательными способностями выбранного пользователя. Длина пути определяется выражением:

$$
\operatorname{len}(P)=\sum_{i=1}^{n-1} W_{i, i+1}, e_{i, j} \in P .
$$

Кратчайшим путем из вершины с в вершину $c^{\prime}$ для графа $W D A C G(s p)$ является такой путь $P$, длина пути которого минимальна [Евстигнеев, 1985]:

$$
P^{*}=\arg \min _{P} \operatorname{len}(P) \text {. }
$$


Определив множества начальных вершин, конечных вершин, можно определить множество всех кратчайших путей (20) из начальных вершин в конечные. Множество данных кратчайших путей и будет являться множеством индивидуальных образовательных траекторий $I L P(u, s p)(21)$ :

$$
\begin{array}{r}
I L P(u, s p)=\left\{P(u, s p, s c, t c) \mid P=\arg \min _{P} \operatorname{len}(P)\right\} \\
\forall s c \in C_{\text {start }}(u, s p), \forall t c \in C_{\text {target }}(u, s p) .
\end{array}
$$

Каждая индивидуальная образовательная траектория учитывает уже изученные дисциплины, целевые курсы и формирует оптимальную для каждого человека последовательность изучения дисциплин. Пример такой последовательности, рекомендуемой для изучения, показан на рисунке 7.

Таким образом, задача поиска оптимального пути изучения дисциплин сводится к задаче поиска кратчайших путей на взвешенном ациклическом ориентированном графе $W D A C G(u, s p)$ между заданными множествами начальных и конечных вершин. Наиболее эффективно задачи нахождения кратчайших путей в графах можно решить при помощи алгоритмов Дейкстры, Беллмана-Форда и других [Левитин, 2006; Томас, 2006].

Можно сформировать алгоритм формирования образовательных траекторий для персонализации порядка изучения дисциплин:

1. Задать множества $S P, S, C, U$;

2. Задать бинарное отношение $\preccurlyeq$, определяющее взаимосвязь дисциплин между собой;

3. Определить матрицу весов $W$, позволяющую управлять связями в графе $W D A C G(s p)$;

4. На основе вышеуказанных множеств, матрицы $W$ и отношения $\preccurlyeq$ сформировать граф $W D A C G(s p)$ выбранного пользователя $u$ и направления подготовки $s p$ сформировать множества $C_{\text {start }}(u, s p)$ и $C_{\text {target }}(u, s p)$;

5. Определить соответствующий подграф $C_{p a t h}(u, s p)$;

6. Вычислить множество $I L P(u, s p)$ при помощи алгоритмов поиска кратчайшего пути на графах.

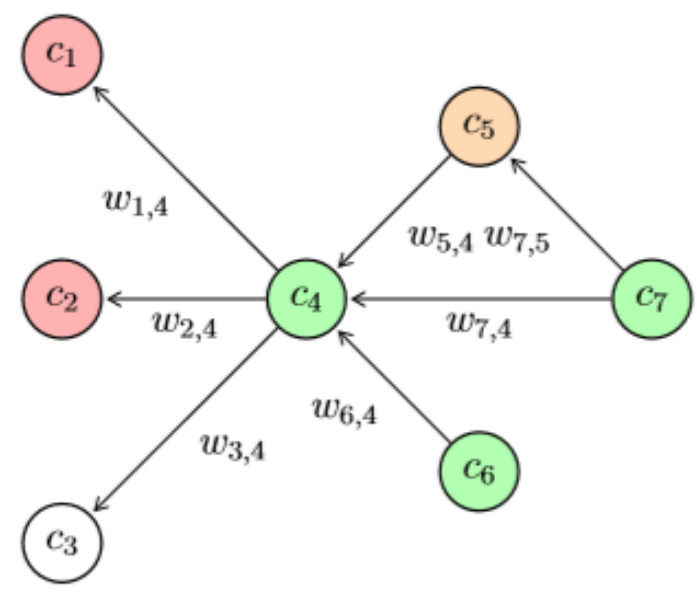

Рис. 7. Пример множества рекомендованных дисциплин

Fig 7. An example of the recommended skills set

В рамках дальнейших исследований предложенная математическая формализация данной концепции будет развиваться и дополняться. В частности, будут исследованы следующие вопросы:

1. Взаимосвязь не только дисциплин, но и компетенций в виде подобного ациклического ориентированного графа. Определение отношения $\preccurlyeq$ при помощи графа компетенций.

2. Определение пользовательских метрик, влияющих на расчет матрицы $W(u, s p)$.

3. Определение метрик компетенций, влияющих на расчет матрицы $W(u, s p)$. Определение влияния работодателей на данные метрики. 
4. Определение метрик дисциплин, влияющих на расчет матрицы $W(u, s p)$.

5. Алгоритмы формирования матрицы $W(u, s p)$. Предполагается использование алгоритмов машинного обучения.

6. Исследование влияния времени на формирование графа $\operatorname{WDACG}(u, s p)$

7. Исследование методов машинного обучения для построения рекомендаций и формирования матрицы $W(u, s p)$.

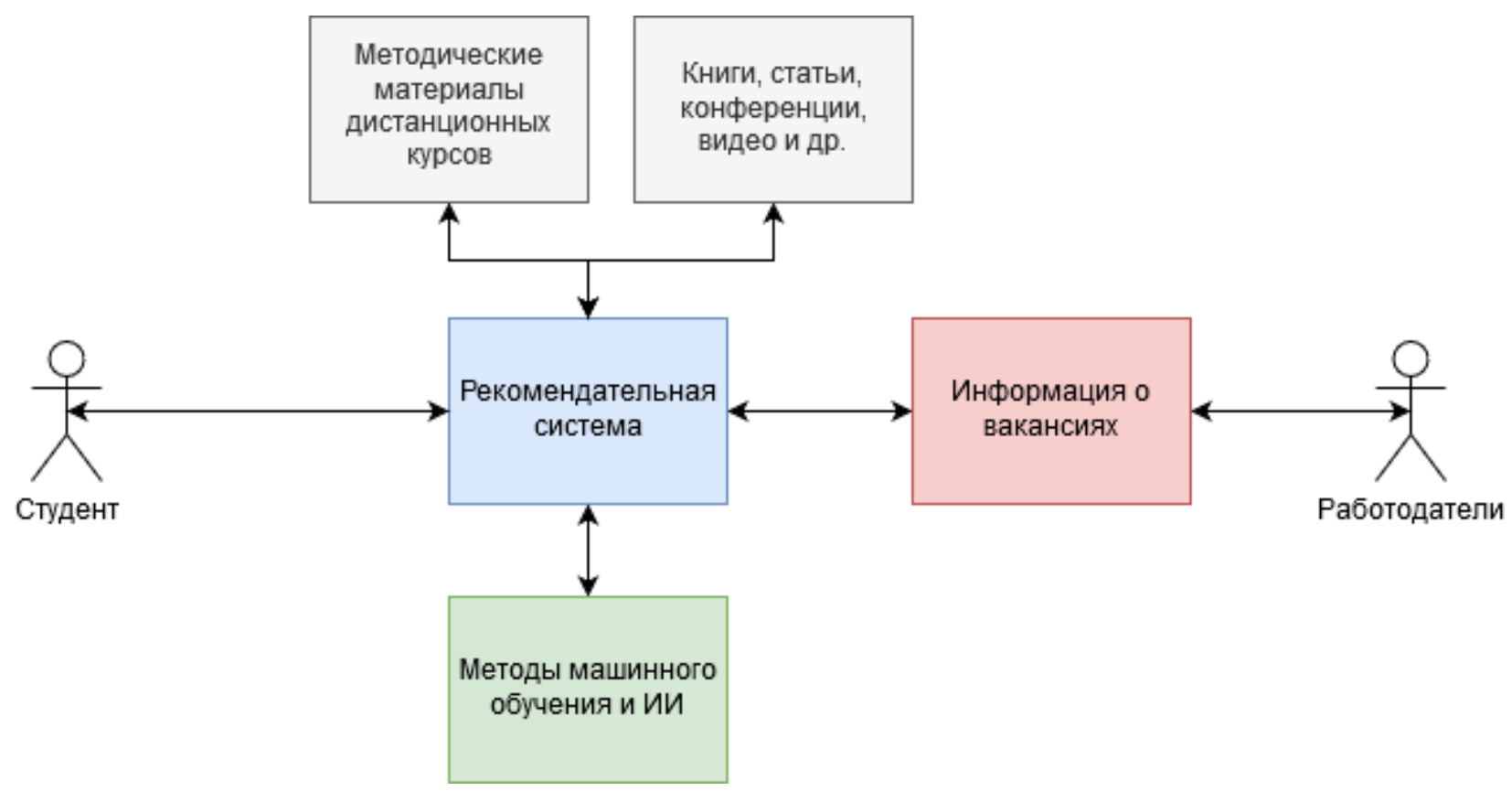

Рис. 8. Структура концепции индивидуальной образовательной экосистемы

Fig. 8. The structure of the concept of the individual education ecosystem

Одним из ключевых понятий в данной математической модели является матрица $W(u, s p)$. Предполагается, что данная матрица не будет задана статически, а будет изменяться в зависимости от различных динамических параметров. На каждом этапе веса в данной матрице будут пересчитываться с использованием методов машинного обучения и построения рекомендаций.

\section{Заключение}

Предложенная в рамках статьи концепция индивидуальной образовательной экосистемы позволит формировать такие персонализированные траектории обучения, которые будут соответствовать как запросам студента, так и запросам работодателей, а также позволят использовать максимально возможное число источников информации для развития компетенций студента.

В дальнейших работах планируется исследование архитектуры и математических методов построения модели ИОЭ, описание сбора данных для ее обучения, формирования метрик и оценочных функций, а также будет произведено экспериментальное исследование и сравнение различных методов машинного обучения для формирования наиболее эффективной реализации концепции индивидуальной образовательной экосистемы.

\section{Список литературы}

1. Алексеев В.Е., Таланов В.А. 2005. Графы. Модели вычислений. Структуры данных. Нижний Новгород: Издательство Нижегородского гос. университета, 307 с.

2. Афанасьев А.Н., Войт Н.Н. 2013. Прогнозирование индивидуальной траектории обучения на основе модели обучаемого с нечёткими характеристиками в автоматизированных системах обучения. Вестник Ульяновского государственного технического университета. 4 (64): 58-61. 
3. Беляев Р.В., Кравец О.Я. 2011. Автоматизация адаптивного управления траекториями обучения. Вестник ВГТУ. № 7. URL: https://cyberleninka.ru/article/n/avtomatizatsiya-adaptivnogoupravleniya-traektoriyami-obucheniya (дата обращения: 30.11.2019).

4. Глущенко А.И. 2009. Разработка метода адаптивного управления обучением по индивидуальной образовательной траектории: автореф. дис. канд. техн. наук. Москва, 19 с.

5. Гримута А.В, Шевченко В.И. Обзор программных систем управления обучением, используемых высшими учебными заведениями. Сборник статей всероссийской студенческой научнотехнической конференции «Мир компьютерных технологий». Севастополь: Севастопольский государственный университет, 2019. С. 224-229. URL: https://elibrary.ru/item.asp?id=41381634 (дата обращения: 20.02.2020)

6. Евстигнеев B.А. 1985. Применение теории графов в программировании. Под ред. А.П. Ершова. Москва: Наука. Главная редакция физико-математической литературы, 352 с.

7. Козлова Е.С., Черкасов А.М., Макашова В.Н., Давлеткиреева Л.3. 2017. Проектирование функциональных возможностей курса системы дистанционного обучения высших школ с учетом индивидуальной траектории обучающихся. International Journal of Open Information Technologies, 50 (4): $78-84$.

8. Кравец О.Я., Заславская О.Ю. 2012. Компетентностная парадигма построения индивидуальной образовательной траектории на основе обратной связи в системе управления обучением. Новый университет. 2 (11): 3-11.

9. Лаврентьев М.А., Шабат Б.В. Методы теории функций комплексного переменного. 4-е изд., М.: Наука, 1972.

10.Левитин А.В. 2006. Алгоритмы. Введение в разработку и анализ. М.: Вильямс, 576 с.

11.Любченко В.В. 2011. Метод будування навчальної траєкторії в умовах мобільного навчання. Вісник Національного технічного університету «Харківський політехнічний інститут»: збірник наукових праць. Тематичний випуск: Інформатика і моделювання. 17:81-85.

12.Мащенко Е.Н., Шевченко В.И., Ченгарь О.В. 2017. Современные информационные технологии в дистанционном образовании. Сборник статей Всероссийской научно-технической конференции «Информационные технологии и информационная безопасность в науке, технике и образовании "ИНФОТЕХ - 2017"» Севастополь: ФГАОУ ВО «Севастопольский государственный университет», C. 116-119. URL: https://elibrary.ru/item.asp?id=32238461 (дата обращения: 20.02.2020)

13.Серебровский В.В., Ткаченко А.В., Ткаченко А.И. 2013. Инновационные технологии в образовании: обучение по индивидуальной траектории. Известия Юго-Западного государственного университета. 1 (46): 26-31.

14.Солдаткин Е.В. 2013. Алгоритм формирования учебной программы на основе композиции нечетких множеств. Cloud of science. № 3. URL: https://cyberleninka.ru/article/n/algoritm-formirovaniyauchebnoy-programmy-na-osnove-kompozitsii-nechetkih-mnozhestv (дата обращения: 30.11.2019).

15.Томас Х. Кормен, Чарльз И. Лейзерсон, Рональд Л. Ривест, Клиффорд Штайн. 2006. Алгоритмы: построение и анализ. Introduction to Algorithms. 2-е изд. М.: «Вильямс», С. 1296.

16.Федеральный закон от 29.12.2012 № 273-Ф3 «Об образовании в Российской Федерации. [Электрон. ресурс]. Режим доступа: http://www.consultant.ru/document/cons_doc_LAW_140174, свободный.

17.Chen C., Duh L. 2008. Personalized web-based tutoring system based on fuzzy item response theory. Expert Systems with Applications, 34(4), 2298-2315.

18.Chen C. 2008. Intelligent web-based learning system with personalized learning path guidance. Computers \& Education, 51 (2), 787-814.

19.Hsu M. 2008. A personalized English learning recommender system for ESL students. Expert Systems with Applications, 34 (1), 683-688.

20.Lin C., Yeh Y.,Hung Y.,Chang R. 2013. Data mining for providing a personalized learning path in creativity: An application of decision trees. Computers \& Education, 68, 199-210.

21.Thulasiraman K.; Swamy M.N.S. 1992, "5.7 Acyclic Directed Graphs", Graphs: Theory and Algorithms, John Wiley and Son, p. 118.

\section{References}

1. Alekseev V.E., Talanov V.A. 2005. Graphs. Computation Models. Data structures. Nizhny Novgorod: Publishing House of the Nizhny Novgorod State. University, 307 p. (in Russian)

2. Afanasyev A.N., Voight N.N. 2013. Prediction of an individual learning path based on a student model with fuzzy characteristics in automated learning systems. Vestnik of the Ulyanovsk State Technical University, 4 (64), 58-61. (in Russian) 
3. Belyaev R.V., Kravets O.Ya. 2011. Automation of adaptive management of learning paths. Vestnik VSTU, No. 7. Retrieved from https://cyberleninka.ru/article/n/avtomatizatsiya-adaptivnogo-upravleniyatraektoriyami-obucheniya. (in Russian)

4. Glushchenko A.I. 2009. Development of a method for adaptive learning management along an individual educational path: abstract of candidate dissertation. Moscow. (in Russian)

5. Grimuta A.V., Shevchenko V.I. 2019. Overview of the learning management software systems used by higher education universities. Collection of articles of the All-Russian Student Scientific and Technical Conference "World of Computer Technology". Sevastopol: Sevastopol State University, P. 224-229. URL: https://elibrary.ru/item.asp?id=41381634 (Accessed: 02.20.2020). (in Russian)

6. Evstigneev V.A. 1985. Application of graph theory in programming. Ed. A.P. Ershov. Moscow: Science. The main edition of the physical and mathematical literature, 352 p. (in Russian)

7. Kozlova E.S., Cherkasov A.M., Makashova V.N., Davletkireeva L.Z. 2017. Designing the functional capabilities of the distance learning system's course of higher schools taking into account the individual trajectory of students. International Journal of Open Information Technologies, 5 (4): 78-84. (in Russian)

8. Kravets O.Ya., Zaslavskaya O.Yu. 2012. Competence paradigm for constructing an individual educational path based on feedback in a learning management system. New University, 2 (11): 3-11. (in Russian)

9. Lavrentiev M.A., Shabat B.V. 1972. Methods of the theory of functions of a complex variable. 4th ed. M.: Nauka, 1. (in Russian) (in Russian)

10.Levitin A.V. 2006. Algorithms. Introduction to the development and analysis. M.: Williams, 576 p.

11.Lyubchenko V.V., Shinkaryuk O.S. 2011. A method of constructing a learning path in the conditions of mobile learning. Vestnik of the National Technical University "Kharkiv Polytechnic Institute": collection of scientific papers. Thematic Issue: Informatics and Modeling, 17: 81-85. (in Russian)

12.Mashchenko E.N., Shevchenko V.I., Chengar O.V. 2017. Modern information technologies in distance education. Collection of articles of the All-Russian Scientific and Technical Conference "Information Technologies and Information Security in Science, Technology and Education "INFOTECH - 2017". Sevastopol: Sevastopol State University, P. 116-119. URL: https://elibrary.ru/item.asp?id=32238461 (Accessed: 02.20.2020). (in Russian)

13.Serebrovsky V.V., Tkachenko A.V., Tkachenko A.I. 2013. Innovative technologies in education: training using an individual path. Proceedings of the Southwestern State University, 1 (46): 26-31. (in Russian)

14.Soldatkin E.V. 2013. The algorithm for the formation of the curriculum based on the composition of fuzzy sets. Cloud of science, No. 3. Retrieved from https://cyberleninka.ru/article/n/algoritm-formirovaniyauchebnoy-programmy-na-osnove-kompozitsii-nechetkih-mnozhestv. (in Russian)

15. Thomas H. Cormen, Charles I. Leiserson, Ronald L. 2006. Rivest, Clifford Stein. Algorithms: construction and analysis. Introduction to Algorithms. 2nd ed. M.: "Williams", p. 1296. (in Russian)

16. Federal Law of December 29, 2012 No. 273-Ф3 On Education in the Russian Federation. [Electronic resource]. Access mode: http://www.consultant.ru/document/cons_doc_LAW_140174, free. (in Russian)

17.Chen C., Duh L. 2008. Personalized web-based tutoring system based on fuzzy item response theory. Expert Systems with Applications, 34 (4), 2298-2315.

18.Chen C. 2008. Intelligent web-based learning system with personalized learning path guidance. Computers \& Education, 51 (2), 787-814.

19.Hsu M. 2008. A personalized English learning recommender system for ESL students. Expert Systems with Applications, 34 (1): 683-688.

20.Lin C., Yeh Y., Hung Y., Chang R. 2013. Data mining for providing a personalized learning path in creativity: An application of decision trees. Computers \& Education, 68, 199-210.

21.Thulasiraman K., Swamy M.N.S. 1992. "5.7 Acyclic Directed Graphs", Graphs: Theory and Algorithms, John Wiley and Son, p. 118

\section{Ссылка для цитирования статьи For citation}

Оболенский Д.М., Шевченко В.И. 2020. Концептуальная модель интеллектуальной образовательной экосистемы. Экономика. Информатика. 47 (2): 390-401. DOI: 10.18413/2687-09322020-47-2-390-401.

Obolensky D.M., Shevchenko V.I. 2020. A conceptual model of the intelligent educational ecosystem. Economics. Information technologies. 47 (2): 390-401 (in Russian). DOI: 10.18413/2687-0932-2020-47-2$390-401$. 